\title{
Primary Diffuse Large B Cell Lymphoma in Kidney with Involvement of Central Nervous System and Heart in a Siamese Cat
}

\author{
Priscylla Tatiana Chalfun Guimarães-Okamoto', Maria Claudia Lopes da Silva', Julio Lopes Sequeira', \\ Alessandre Hataka', Fernanda Chicharo Chacar'2, Silvano Salgueiro Geraldes', \\ José Francisco Antunes Ribeiro' ${ }^{1}$ \& Alessandra Melchert'
}

\begin{abstract}
Background: Lymphoma is the most common neoplasia in cats and usually happens in middle-aged males of European breeds, FeLV-positive test is also observed in $25 \%$ to $50 \%$ of these cases. The diagnostic of renal lymphoma is based on clinical signs, the diagnosis is based on clinical signs associated with additional tests. Histological and immunohistochemical evaluations allows the classification which can provide prognostic information. Central nervous system metastasis is frequently reported, but lingual and lung lobe extension were also reported. Here, we report a case of renal lymphoma with systemic involvement of heart and CNS in a cat.

Case: A 3-year-old male Siamese cat presented with weight loss, emesis, anorexia, polydipsia and polyuria for at least 3 weeks. The clinical parameters were stable, but presenting pale mucosal membrane and also severe dehydration. Complete blood count and chemistry profile revealed anemia and severe azotemia. On abdominal palpation, bilateral renomegaly and irregular contours of both kidneys were also detected. The kidneys were enlarged and had irregular contours on palpation, confirmed by abdominal ultrasonography, that revealed kidneys with markedly irregular contours, and the presence of fluid with high cellularity between cortical and renal capsule (capsular fluid). An aspiration citology by fine-needle from the capsular fluid guided by ultrasound was collected and sent to cytological analysis, which suggested lymphoma. The aspirate was moderately cellular and consisted of large round cells with distinct, scant, basophilic cytoplasm, round nuclei with dense chromatin, inconspicuous nucleoli, and large amount of naked cells. On the second day of treatment the cat presented a cardiopulmonary arrest not responsive to cardiopulmonary cerebral resuscitation, died, and the owner authorized the necropsy, allowing the renal biopsy showing that the same neoplastic cells presented in kidneys were also seen in heart and central nervous system, suggesting the occurrence of primary renal lymphoma with involvement of to these tissues, and the diagnosis was high grade diffuse large cell lymphoma. And also histopathological exam diagnosed high grade diffuse large cell lymphoma.

Discussion: The patient died, and the post-mortem histological analysis confirmed the diagnosis of primary diffuse large B cell lymphoma in kidney with systemic involvement of heart and central nervous system (CNS). Lymphoma is the most common renal tumor in cats, however primary feline renal tumors are rare and few reports are available at this time. Here we report the occurrence of primary renal lymphoma in a Siamese cat, and there's no previous report of this type of lymphoma especially in this breed. More investigation is needed to evaluate the susceptibility of Siamese cat breed to the development of primary renal lymphoma. It is difficult to determine whether the renal involvement is primary or it is a consequence of the multicenter type. In the present report the same neoplasic infiltration observed in kidney was also found in central nervous system and heart. The cytology is a quickly, non-invasive, and sensitivity method for the diagnostic of lymphoma. However, the cytology do not provides the immune diagnosis of diffuse large B cell, and although it is useful for staging lymphoma, histopathology and immunohistochemistry is always necessary for classification and grading. Since the diagnosis, the cat of this report survived only 2 days, which reinforces the bad prognosis of renal lymphoma. The renal lymphoma should be considered as a potential cause of severe azotemia, and should be taken as the mainly differential diagnosis among neoplasias of kidneys in cats.
\end{abstract}

Keywords: oncology, feline, extranodal, lymph nodes, cancer. 


\section{INTRODUCTION}

Lymphoma is the most common neoplasia in cats, comprising approximately $33 \%$ of all tumors in this specie $[7,11,14]$. On the other hand, primary renal tumors are rare, accounting almost $1.5 \%$ of all feline neoplasms $[6,11,13]$. However, renal lymphoma is the most common primary tumor of kidney in cats, and usually happens in middle-aged males of European breeds. FeLV-positive test is also observed in $25 \%$ to $50 \%$ of these cases $[8,12]$. The diagnostic of renal lymphoma is based on clinical signs, such as weight loss, emesis, polyuria and polydipsia, complete blood count, chemistry profile, mainly BUN (Blood urea nitrogen) and creatinine, because patients usually present renal insufficiency, abdominal ultrassonography, cytology and histopathologycal findings. Although both cytology and histopathology may be satisfactory for the diagnosis of lymphoma, histological and immunohistochemical evaluations allows the classification of lymphoma into morphologic subtypes, which can provide prognostic information $[5,10,14]$. Dissemination to central nervous system is frequently reported in cases of renal lymphoma, but lingual and lung lobe extension were also reported [2,3]. Here, we report a case of renal lymphoma with systemic involvement of heart and CNS in a cat.

\section{CASE}

A 3-year-old $3.0 \mathrm{~kg}$, intact male, indoor-only Siamese cat was referred for a 3 week history of weight loss, emesis, anorexia, polydipsia and polyuria. Upon this presentation, clinical parameters such as temperature, heart rate, breath, pulse, capillary refill time and palpable lymph nodes were normal, except by the mucosal membrane that was pale, and the patient was also severely dehydrated. On abdominal palpation, bilateral renomegaly and irregular contours of both kidneys were detected.

Complete blood count and chemistry profile revealed anemia (Haematocrit $=17 \%$ ) and severe azotemia (creatinine $=8.5 \mathrm{mg} / \mathrm{dL}, \mathrm{BUN}=170 \mathrm{mg} / \mathrm{dL}$ ), respectively. Abdominal ultrasonography detected bilateral asymmetrical renomegaly, changes in renal morphology, no preservation of corticomedullary differentiation, increased medullary renal echogenicity, and the presence of fluid with high cellularity between cortical and renal capsule (capsular fluid). Color Doppler revealed distortion of intraparenchymal renal vasculature. Spectral Doppler detected incresead levels of resistivity of both interlobar and renal arteries, which ranged from 0.78 to 0.83 (normal range $<0.70$ ). Intraparenchymal vascularization was detected in systolic peaks elevated (above $4 \mathrm{KHz}$ ) which has been highly indicative of malignancy in humans. The measurements of the Doppler velocity values were not possible.

The cat was sedated with morphine sulfate (Dimorf $\left.^{\circledR}\right)^{1}$ and acepromazine maleate $\left(\text { Apromazin }{ }^{\circledR}\right)^{2}$ and a fine-needle aspiration sample guided by ultrasound from the capsular fluid was collected and sent to cytological analysis. The aspirate was moderately cellular and consisted of large round cells with distinct, scant, basophilic cytoplasm, round nuclei with dense chromatin, inconspicuous nucleoli, and large amount of naked cells. The cytologic diagnosis was probable lymphoma.

The clinical condition of the patient did not allow the performance of kidney biopsy at that time. Intravenous fluidotherapy, enteral nutrition approach and antiemetic medication were provided as support therapy, but the animal remained worsening. On the second day of treatment the cat presented a cardiopulmonary arrest not responsive to cardiopulmonary cerebral resuscitation, and died. The owner authorized the necropsy.

On postmortem examination, both kidneys were enlarged and spherical masses protruded from the renal surface. On cut section, the mass was located on the cortex, infiltrating pelvis, and it was composed of a homogenous, firm, white to yellow tissue. The renal medulla was preserved.

The histopathological examination of kidneys demonstrated large round cells with acidophilic cytoplasm varying from scant to abundant, round nuclei with dense chromatin, and prominent, single nucleoli. There was also marked anisokaryosis and anisocytosis. The cells were arranged in sheets, with scarce fibrous stroma. Rare areas of mineralization in the renal tubules were noted (Figure 1A).

The same type of cells was found interspersed in heart muscle fibers, and it was also observed in cerebellum, meninges and cerebral cortex. The histopathological exam showed that the same neoplastic cells presented in kidneys were also seen in heart and central nervous system, suggesting the occurrence of primary renal lymphoma with involvement of to these tissues. The diagnosis was high grade diffuse large cell lymphoma.

Immunohistochemestry was performed and the neoplastic tissue of kidney was assessed for the expression of $\left(\mathrm{CD} 20^{\circledR}\right)^{3}\left(1: 1000\right.$, policlonal) and $\left(\mathrm{CD}^{\circledR}\right)^{4}$ [1:500, policlonal] for pan B-cell and T-cell respectively. The result of CD20 positive diffuse membrane expression on the neoplastic cells (Figure 1B) and negative expression to $\mathrm{CD} 3$ (Figure 1C) established the final diagnosis of primary diffuse large B cell lymphoma in kidney. 


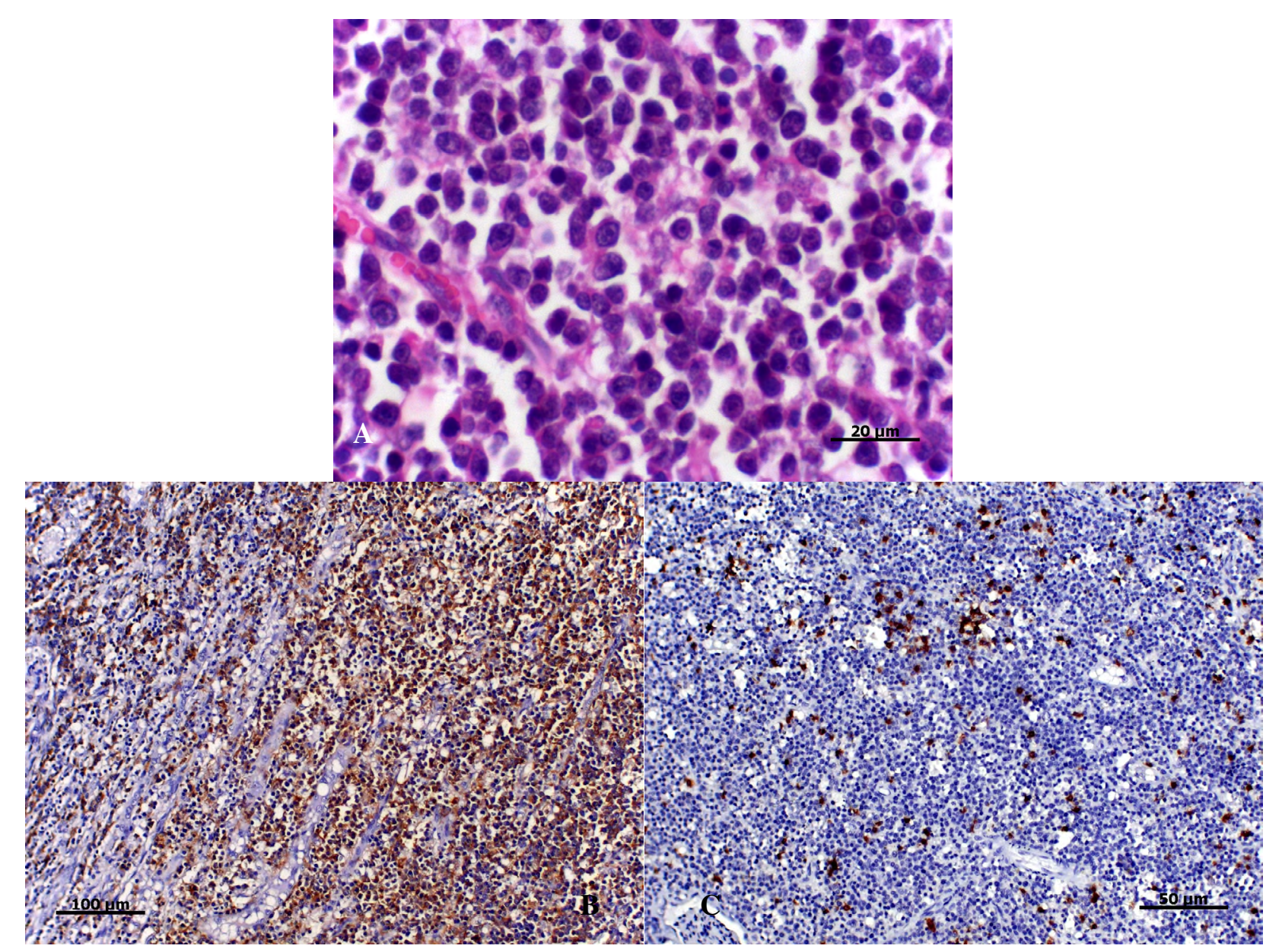

Figure 1. A- High grade large cell lymphoma [HE, 40x objective]. B- Large B cell lymphoma. CD20 positive immunoassay by neoplastic cells [20x objective]. C- CD3 negative immunoassay by neoplastic cells [20x objective].

\section{DISCUSSION}

Renal lymphoma is the most common primary neoplasm in kidney of cats. It usually affects male on middle-age or older and FeLV negative cats [10]. On the other hand, in one Australian study $50 \%$ of cats with naturally-occurring lymphoma were positive for FIV antibodies, and these animals had a medium age of 9 years. In this same study, almost $54 \%$ of the cats presented with renal lymphoma were FIV-positive [7]. Here, we report a 3-year-old cat, a relatively young animal, then we hypothesized that the cat was FeLVpositive. We do not suggest that the cat was also FIVpositive because it was a single only-indoor pet, and FIV infection is most common in feral, outdoor, intact male cats. Biting associated with territorial aggression is the most important form of virus transmission, and free-roaming, entire, male cats are at increased risk to be infected by FIV [1]. However, the FIV/FeLV test was not performed because the owner had some retrenchment of costs, and it is for sure a limitation of our report.

European breeds are considered to be predisposed to develop renal lymphoma, and there are some reports that cite the predisposition of Domestic Shorthair too, but not Oriental breeds such as Siamese or Oriental [12]. Siamese is known to be genetically predisposed to mediastinal lymphoma but no information is available yet about Siamese breed predisposition to other types of lymphoma [7,9]. Here we report the occurrence of primary renal lymphoma in a Siamese cat, and there's no previous report of this type of lymphoma especially in this breed. More investigation is needed to evaluate the susceptibility of Siamese cat breed to the development of primary renal lymphoma.

Although lymphoma is the most common tumor of kidney in cats, in most part of the cases it is not possible to determine whether the renal involvement is primary or it is a consequence of the multicenter 
type $[4,8]$. In the present report the same neoplasic infiltration observed in kidney was also found in central nervous system and heart, and it was not seen in any of the others tissues, which were normal. We could conclude that the cat really had a primary large B cell lymphoma in kidney with involvement of central nervous system and heart.

The cytology is a quickly, non-invasive, and sensitivity method for the diagnostic of lymphoma. However, the cytology do not provides the immune diagnosis of diffuse large B cell, and although it is useful for staging lymphoma, histopathology and immunohistochemistry is always necessary for classification and grading [5,14]. In the present report, the cytology exam from the capsular fluid suggested lymphoma, which was later confirmed by the histopathology of the kidneys with a high grade large B cell lymphoma.
The rate of survival in cases of renal lymphoma is shorter than other types of this neoplasia, due to its invasive and aggressive character, and the survival can range from 3 to 6 months [2]. Since the diagnosis, the cat of this report survived only 2 days, which reinforces the bad prognosis of renal lymphoma.

The renal lymphoma should be considered as a potential cause of severe azotemia, and should be taken as the mainly differential diagnosis among neoplasias of kidneys in cats.

\section{MANUFACTURERS}

${ }^{1}$ Cristália Produtos Químicos Farmacêuticos. Itapira, SP, Brazil.

${ }^{2}$ Laboratório Rhobifarma. Hortolândia, SP, Brazil.

${ }^{3}$ SpringBioscience. Pleasanton, CA, USA.

${ }^{4}$ Dako do Brasil Ltda. São Paulo, SP, Brazil.

Declaration of interest. The authors report no conflicts of interest. The authors alone are responsible for the content and writing of the paper.

\section{REFERENCES}

1 Beatty J. 2014. Viral causes of feline lymphoma: Retroviruses and beyond. The Veterinary Journal. 201(2): 174-180.

2 Bound N.J., Priestnall S.L. \& Cariou M.P. 2011. Lingual and renal lymphoma in a cat. Journal of Feline Medicine and Surgery. 13(4): 272-275.

3 Brown A.L., Beatty J.A. \& Nicoll R.G. 2011. Dyspnoea and pulmonary consolidation in a cat with T-cell lymphoma. Journal of Feline Medicine and Surgery. 13(10): 772-775.

4 Bryan J.N., Henry C.J., Turnquist S.E., Tyler J.W., Liptak J.M., Rizzo S.A., Sfiligoi G., Steinber S.J., Smith A.N. \& Jackson T. 2006. Primary renal neoplasia of dogs. Journal of Veterinary Internal Medicine. 20(5): 1155-1160.

5 Burkhard M.J. \& Bienzle D. 2013. Making sense of lymphoma diagnostics in small animal patients. Veterinary Clinics of North America: Small Animal Practice. 43(6): 1331-1347.

6 Chew D.J., DiBartola S.P. \& Schenk P. 2010. Tumors of the urinary system. In: Chew D.J., DiBartola S.P. \& Schenk P. (Eds). Canine and Feline Nephrology and Urology. 2nd edn. St. Louis: Saunders Elsevier, pp.434-464.

7 Gabor L.J., Love D.N., Malik R. \& Canfield P.J. 2001. Feline immunodeficiency virus status of Australian cats with lymphosarcoma. Australian Veterinary Journal. 79(8): 540-545.

8 Henry C.J., Turnquist S.E., Smith A., Graham J.C., Thamm D.H., Brien M.O. \& Clifford C.A. 1999. Primary renal tumours in cats. Journal of Feline Medicine and Surgery. 1(3): 165-170.

9 Lorimer H.E. 1999. Hereditary lymphosarcoma in Oriental Shorthair cats. Journal of Feline Medicine and Surgery. (Special Supplement). p.29.

10 Moore A. 2013. Extranodal lymphoma in the cat - Prognostic factors and treatment options. Journal of Feline Medicine and Surgery. 15(5): 379-390.

11 Schmidt S., Nerschbach V., Eberle N., Mischke R., Nolte I. \& Betz D. 2012. Das renale Lymphom bei der katze: diagnostik, therapieverlauf und überlebenszeit - ein fallbericht. Tierärztliche Praxis Kleintiere. 40(4): 271-277.

12 Seguela J., Layssol-Lamour C., Geffre A., Trumel C., Raymond-Letron I. \& Lanore D. 2008. Cas clinique: lymphome rénal traité par chimiothérapie chez un chat. Revue Médecine Vétérinaire. 159(4): 192-197.

13 Taylor S.S., Goodfellow M.R., Browne W.J., Walding B., Murphy S., Tzannes S., Gerou-Ferriani M., Schwartz A. \& Dobson J.M. 2009. Feline extranodal lymphoma: response to chemotherapy and survival in 110 cats. Journal of Small Animal Practice. 50(11): 584-592.

14 Twomey L.N. \& Alleman A.R. 2005. Cytodiagnosis of feline lymphoma. Compendium on Continuing Education for the Practising Veterinarian. 27(1): 17-31. 